\title{
Study on shock failure characteristics of gas explosion in coal mines
}

\begin{abstract}
Based on the mechanism and propagation characteristics of gas explosion, the influencing factors of gas explosion propagation in coal mines were analyzed, which include methane concentration, methane pressure, initial temperature, inert gas, ignition source, oxygen concentration, explosion space characteristics, and external environment conditions. Different factors play different roles in different stages and different states. Gas explosion in coal mines can cause serious destruction; the injury effect on personnel is mainly manifested in three aspects: the killing effect of shock wave overpressure (impulse) on personnel; the injury caused by dynamic pressure; the personnel asphyxiation effect caused by toxic and harmful gases. The three kinds of personnel injury effect act individually or simultaneously. The shock wave failure can be measured by three characteristic parameters: peak overpressure, impulse and duration time. The shock wave failure criterion includes the overpressure criterion, the impulse criterion and overpressure-impulse criterion; and the overpressure criterion is most commonly used. Finally, the reflection tension failure mechanism of shock wave and the failure mechanism of the high-temperature high-pressure gas were summarized; the failure process of gas explosion in coal mines was obtained.
\end{abstract}

Volume 3 Issue 3 - 2018

\author{
Zhenzhen JIA, Qing YE, Yi LU \\ School of Resource, Hunan University of Science and \\ Technology, China
}

Correspondence: Zhenzhen JIA, School of Resource, Environment and Safety Engineering, Hunan University of Science and Technology, Xiangtan 4II201, China, Email jiazhenzhen I982@।26.com

Received: November 29, 2017| Published: May 032018

Keywords: gas explosion, shock wave, failure mechanism, reflection tension failure, high-temperature high-pressure gas

\section{Introduction}

Explosion is the radiation process that the system energy releases suddenly within finite space or rapidly transforms into kinetic energy, mechanical energy, and heat. Since gas from coal and rock is emitted and accumulates within a finite space, gas explosion in coal mines is a kind of non-point source explosion. The characteristics of gas explosion and condensed explosive have great difference, for gas explosion strongly depends on environmental conditions. The process of gas explosion involves turbulent flow, heat and mass transfer, chemical reaction, and the coupling interaction between flame and pressure wave. The significant feature of this process is shock wave caused by gas explosion, which can make considerable damage. Generally speaking, flame is constantly accelerated during the propagation process, and shock wave and even detonation can be generated under certain conditions, thus bringing greater damage. When shock wave caused by gas explosion impacts roadways, the stability and security of underground engineering within a certain range near explosion sources are usually endangered. When the flame speed is less than $100 \mathrm{~m} / \mathrm{s}$, shock wave can be approximately regarded as sound wave and will cause slight structural damage. When the flame speed reaches $200 \mathrm{~m} / \mathrm{s}$, the severe turbulence effect can be caused; the failure effect of explosion wave caused by pressure wave of high-speed flame is similar to the failure effect caused by detonation wave, they both bring severe structure damage and the failure range can reach thousands of meters. ${ }^{1,2}$ In some coal mines, shock wave generated by gas explosion can even destroy the buildings and people on the ground through wellhead. According to time, explosion process can be divided into two stages: the gas ignition and the gas explosion propagation. Gas explosion brings great destruction mainly during gas explosion propagation, and flame and shock wave are the main factors of damage. Experimental research for years and scene investigation of gas explosion accidents for many times show that the deadly risk factors caused by gas explosion are: overpressure damage and action time of shock wave, heat radiation, high temperature burning, toxic and harmful gases, and asphyxiation.,

\section{Influencing factors of gas explosion in coal mines}

The influencing factors of gas explosion can be divided into two aspects: internal factors and external factors. Internal factors include: methane concentration, ignition source, and oxygen concentration, and etc. The external factors are external environmental conditions. ${ }^{5}$

\section{Methane concentration}

According to the theory of chain reaction, methane with a certain concentration decomposes a mass of activation centers after absorbing adequate heat, and completes the entire oxidation process with releasing a certain quantity of heat. If heat generated by the chain reaction is more than that absorbed by ambient media, and there is adequate methane and oxygen in the mixture; more activation centers will be generated, thus making oxidation process develop into explosion rapidly. Therefore, gas explosion happens at a certain range of methane concentration which we call the methane explosion limits. In fresh air, the methane explosion limits are from 5\% to $16 \%$, and its equivalent concentration is $9.5 \%$; therefore, when methane concentration in the methane-air mixture is lower than the lower limit or higher than the upper limit, the contact between the mixture and ignition sources will not cause flame to spread by itself. When methane concentration in the methane-air mixture is lower than the lower limit; the flame propagation is impeded, because excess air as the inert medium participates in the combustion reaction and consumes part of reaction heat. When methane concentration in the methane- 
air mixture is higher than the upper limit; the flame propagation is also impeded, because excess methane and insufficient oxygen results in incomplete chemical reaction, making the heat released in the reaction less than the heat loss. The methane explosion limits are not static; they are affected by many factors, for example: the mixing of combustible gases, the mixing of coal dust, the mixing of inert gases, the initial temperature of the mixed gas, humidity, ignition sources, and etc. The methane concentration is one of the key factors affecting gas explosion reaction, which directly affects the reaction speed and the highest temperature of flame. When methane concentration reaches the equivalent concentration, the reaction is fastest and the explosion is strongest.

\section{Initial pressure}

For coal mine gases, the environmental pressure has some effect on the explosion limits of methane and other hydrocarbons. But in general, it has little impact on the lower limit, while much impact on the upper limit. When the system pressure increases; the explosion limits extend and the upper limit rises more significantly. This is because the distance between molecules shortens and the collision probability increases with the increase of pressure, thus making the occurrence and development of flame more easily. When the system pressure reduces, the distance between molecules widens and the range of explosion limits gets smaller. When the pressure drops to a certain value (i.e. critical value), the upper explosion limit equals to the lower explosion limit. If the pressure drops continuously, the system will become non-combustible and non-explosive. Therefore, the operation under negative pressure in a closed vessel is beneficial for safety production. ${ }^{6}$

\section{Initial temperature}

In the air, the explosion is most likely to occur when the methane concentration is between $4.9 \%$ and $15.4 \%$, the combustion is most likely to occur at a methane concentration of $8 \%$, and the explosion is most violent at a methane concentration of $9.5 \%$. The lower explosion limit of methane-air mixture reduces with increasing temperature, and the lower explosive limit is $3 \%$ at a temperature of $400^{\circ} \mathrm{C}$. When the methane-air mixture contacts with ignition sources at a temperature between $700^{\circ} \mathrm{C}$ and $800^{\circ} \mathrm{C}$; the methane-air mixture cannot burn or explode immediately, but it can be ignited after a period of time, which is called the induction period. This is because the specific heat capacity of methane is large, the methane can decompose and combust after absorbing a certain quantity of heat. When the pressure is constant, the induction period mainly depends on the methane concentration and the temperature of ignition sources. Under normal circumstances, within the explosion limits, the higher the methane concentration is, the longer the induction period will be; the higher the temperature of ignition source is, the shorter the induction period will be. The induction period is $10 \mathrm{~s}$ at a temperature of $650^{\circ} \mathrm{C}$; the induction period is $1 \mathrm{~s}$ at a temperature of $1000{ }^{\circ} \mathrm{C}$. Consequently, the explosion limits are not static; they are affected by the factors such as initial temperature of methane-air mixture. Under the complex conditions in coal mines, the change of the explosion limits cannot be ignored.

\section{Inert gas}

The range of explosion limits gets smaller with adding inert gas into the methane-air mixture. When the concentration of inert gas increases to a certain value, the mixture will become non-combustible and non-explosive.

\section{Oxygen concentration}

The explosion can occur only when the methane concentration of the methane-air mixture is within the methane explosion limits, and the gas explosion limits get narrow with reducing the oxygen concentration. When the oxygen concentration decreases; the lower limit of methane explosion increases slowly and the upper limit drops rapidly. When the oxygen concentration decreases to $12 \%$, the methane-air mixture will not explode even contacting with ignition source; that is to say, $12 \%$ is the oxygen concentration out of explosion. Accordingly, the control for oxygen concentration is very important to prevent gas explosion in fire zones.

\section{Ignition source}

The ignition source of gas explosion in coal mines can be divided into four categories: spark ignition, heat ignition, flame ignition and mechanical spark ignition; their ignition mechanism have great difference. Spark ignition can be regarded as the process that the underground electrical equipment discharges to excitation a certain space range of flammable gas molecules, thus obtaining the essential activation energy. Heat ignition can be described to be heat flux of a sudden influx in premixed gas space. Flame ignition can be regarded to be the results caused by other combustion reactions which occur suddenly in the underground space containing gas. The mechanical spark ignition can be described by the model of spark ignition, but its mechanism is more complex, such as the mechanical spark caused by friction or collision. The lowest temperature of methane ignition is between $650^{\circ} \mathrm{C}$ and $750{ }^{\circ} \mathrm{C}$. The different concentration of methane has different ignition temperature. Currently, combustion is considered to be the most likely to occur when the methane concentration is between $7 \%$ and $8 \%$. In standard conditions, the minimum ignition energy is $0.28 \mathrm{~mJ}$; only when the energy of ignition source is more than the minimum ignition energy, the combustion or explosion of methane can occur. The ignition position and ignition intensity have important influence on the development of gas explosion. The ignition at the closed end of blind galley usually brings higher overpressure and faster combustion speed, for example, gas explosion in the tunneling working face brings more serious destroy than that in other places. The influence of ignition intensity on gas explosion is verified from experiments, and it is found that ignition source with high intensity, such as detonator and dynamite, can directly cause explosion and even detonation under the same conditions. The ignition of gas explosion in coal mines is weak ignition, and the electric spark ignition is usually used in experiments.

\section{Explosion space characteristics}

The finite space (or container) where the explosion source exists has effect on gas explosion. Experiments have proved that the smaller the diameter of explosion space (or container) is, the narrower the explosion range will be. This can be explained by heat transfer and wall effect. According to heat transfer, gas per unit volume has more heat loss on the wall with reducing the diameter of container (or pipe). Some literatures report that the flame will go out when the heat released by container (or pipe) is equal to $23 \%$ of the energy released by flame; therefore, the increase of heat loss will reduce the flame propagation speed and affect the explosion limits. According to wall effect, the condition for the persistence of combustion is that the number of newborn free radicals is equal to or is more than the number of free radicals consumed. Therefore, with reducing the diameter of container (or pipe); the collision probability between free radicals 
and reaction molecules is decreasing, while the collision probability between free radicals and the wall is increasing. When the diameter of container (or pipe) reduces to a certain value, the combustion cannot go on as s result of wall effect.

External environment conditions: The environmental conditions of gas explosion in the underground roadway are complex; the influence of environmental conditions on gas explosion mainly contains the roadway size, roadway structure form, obstacles in the roadway, coal dust, and etc. ${ }^{6,7}$

1. The roadway size: The underground roadway system is built for production and ventilation, the section area is large. The experimental results of gas explosion in pipes with a smaller size have some difference from the results of gas explosion in actual roadways.

2. The roadway structure form: The roadway structure form includes: bends in the roadway, branches of roadway, change of cross section, cross section shape, and etc. These factors have a significant impact on gas explosion process, for example, bends in the roadway can make overpressure at local spots increase greatly and pressure wave reflected. Other factors except the above factors have little influence on gas explosion, such as the roadway shape.

3. The obstacles in roadway: There are obstacles in the underground roadways, roadway supports, machinery-transport equipment, ventilation equipment, and etc. are the most common obstacles. The distribution of these obstacles is usually irregular, but it can be simplified as regular distribution in numerical simulation and experimental research. Experimental research and field investigation find that obstacles can promote gas explosion and its propagation.

4. The coal dust in roadway: When gas explosion occurs; coal dust is blown up by the precursor shock waves in, spreading in the air, and may participate in gas explosion, which makes significant effect on gas explosion propagation.

\section{Shock failure mechanism of gas explosion}

Theoretical analysis and field accident survey ${ }^{5,8}$ show that the injury on personnel caused by gas explosion is mainly manifested in three aspects: the killing effect of shock wave overpressure (impulse) on personnel; the injury caused by dynamic pressure; the personnel asphyxiation effect caused by toxic and harmful gases. The three kinds of personnel injury effect act individually or simultaneously, which depends on gas explosion and the position of disaster area personnel. For gas explosion in blind roadway, the dynamic pressure impaction of shock wave and asphyxiation of toxic and harmful gases are relatively small, thus overpressure (impulse) of shock wave is mainly considered to measure damage caused by gas explosion. The damage of shock wave overpressure can be described by three characteristic parameters: peak overpressure, impulse and duration time. The failure criterion of shock wave includes the overpressure criterion, the impulse criterion and overpressure-impulse criterion; and the overpressure criterion is most commonly used. The overpressure on shock wave front is related to the energy generated by shock wave. Under the same conditions, the higher the energy of gas explosion is, the higher the intensity of shock wave and the overpressure on the shock wave front will be. Accordingly, much attention is paid to how to attenuate the intensity and impulse of shock wave.
After gas explosion occurs, the methane-air mixture almost instantaneously changes into high-temperature high-pressure gas, namely explosion products. The high-temperature high-pressure gas expands rapidly, forcing the air around it to leave the original position and move outward rapidly; consequently, a compressed air layer is generated at the forefront of the high-temperature high-pressure gas, i.e. shock wave. The shock wave pressure depends on the energy released by gas explosion and the distance from explosion source. In fact, almost all energy produced by gas explosion transforms into the energy of explosion wave, explosion products spread backward along the explosion wave front at a high speed. With the increase of the propagation distance and the friction between the gas and roadway wall, the average energy flow density of gas per unit mass is decreasing. The average energy decreasing and the energy loss during propagation process make the propagation speed of explosion products reduce quickly to zero, namely forming the pressure decreasing zone. The volume is expanding with the decrease of pressure. When explosion products expand to a particular volume; the pressure will reduce to the initial pressure of ambient air without disturbance, while explosion products will not stop moving but keep on expanding to a maximum volume due to inertia. At this time, the average pressure of explosion products is lower than the initial pressure of ambient air without disturbance, forming the negative pressure zone near explosion source. After negative pressure zone appears; the ambient air in turn moves to explosion center, compresses explosion products for the first time, making the pressure of explosion products increasing. Similarly, when the pressure of explosion products equals to the initial pressure of ambient air without disturbance; the compression of ambient air on explosion products will not stop but keep on due to inertia, making the pressure of explosion products greater than the initial pressure. And then the second expansion-compression pulse process begins. After several expansion-compression processes, the pulse process stops with achieving system equilibrium. Since the density and inertia of $\mathrm{CH} 4$ are small, only the first expand-compression pulse process has a practical significance. Therefore, shock wave can be divided into two parts: the first part following shock wave with an overpressure greater than zero (namely positive pressure zone) and the second part with an overpressure less than zero (namely negative pressure zone).

Accordingly, in actual explosion-proof design, we should not only pay attention to the first impact of gas explosion (namely positive impact), but also pay attention to the second reverse impact when the explosion from the periphery rushes back to the explosion source. The pressure of the second reverse impact is smaller than positive impact pressure; but the second reverse impact can cause more damage, because the second reverse impact is based on damaged roadways. If methane and coal-dust exist in explosion hazardous area, the second reverse is likely to cause the second explosion, bringing more serious destroy. When explosion wave encounters objects; the process is very complex, including reflection, diffraction, and etc. Its damage types vary with the pressure on objects, which mainly depend on overpressure of shock wave and impulse of positive pressure zone. The dynamic effect of wall mainly depends on the impulse of explosion which is the pressure of explosion on wall. Theory and practice indicate that overpressure and impulse are important parameters for particular failure modes or damage levels. The overpressure-impulse criterion indicates that damage depends on the overpressure and impulse. Various types of damage are very complex and have a great relationship with the shape, position and orientation of objects. 


\section{Reflection tension failure analysis of shock wave}

On the basis of the existing achievements, Hino Kumaxiong, a Japanese scholar, systematically proposed the theory of shock wave tension failure in $1953 .{ }^{9}$ When gas explosion occurs in underground roadways in coal mines; the shock wave with high temperature, high pressure and high velocity is generated instantaneously and spreads outward from the explosion point. The intensity of shock wave greatly exceeds the dynamic compressive strength of the roadway wall, thus causing the coal and rock of roadway wall broken and forming crush zone. When shock wave is attenuated into compression stress wave; the compression stress and the compression deformation in the radial direction on the roadway surface are generated, the tension stress and tension deformation in the tangential direction on the roadway surface are generated. In addition to that the tensile strength of coal and rock is far less than its compressive strength; consequently, the crack zone of coal and rock will be formed. After the compression stress wave spreads through the crack zone, its intensity greatly reduces so that it cannot directly make coal and rock crushed. When the compression stress wave gets to the free surface, it is reflected and the tension wave is formed, which spreads from free surface to roadway wall. The intensity of the compression wave has greatly decreased, but the tension fracture of the coal and rock still occurs as a result of that the tension intensity of coal and rock is far less than its compressive intensity. With the reflection wave spreading, the cracks caused by layer spallation and stress wave intersect, until the coal and rock within damage range completely breaks. Therefore, shock wave of gas explosion plays a major role in breaking the coal and rock of roadways; it not only affects the development of roadway wall cracks, but also creates the conditions for the subsequent process of gas explosion. Thus shock wave of gas explosion plays a dominant role on wall broken in the explosion area and has important influence on the stability and security of the roadway away from the explosion area.

\section{Failure mechanism of high temperature high pressure gas}

U. Lange Fuchs, a Swedish scholar, presented the expansion failure theory of explosion gas in 1963. According to the theory, the expansion of explosion gas is the main reason for breaking the coal or rock. When gas explosion takes place in the underground; the explosion shock wave firstly activates the original micro cracks in the coal and rock around the explosion zone, and then the subsequent shock wave makes these cracks develop further and the reflected tension wave on the free surface is generated, leading to damage. The flame front with violent chemical reaction follows the shock wave, the temperature of which is between $185^{\circ} \mathrm{C}$ and $2650^{\circ} \mathrm{C}$; thus the energy caused by gas explosion can generate high-temperature highpressure gas. When the high-temperature high-pressure gas acts on the roadway surface, it diffuses into the initial radial cracks caused by explosion shock wave (or preexisting fissures), thus expanding the cracks and forming tension stress field in the tangential direction. The quasi-static stress field and gas compression make cracks extend forward. When the free surface is nearby; the cracks extends to the free surface, making it broken. The high-temperature high-pressure gas is used to wedge into the cracks, makes cracks intersect and break coal into blocks, and throws these broken blocks. The energy released by explosive mixed gas can be directly calculated by the quantity and combustion heat (i.e. high calorific value) of combustible gases in the reaction.

\section{Conclusion}

1. The influencing factors of gas explosion propagation in coal mines were analyzed; and then we found that the influencing factors include: methane concentration, methane pressure, initial temperature, inert gas, ignition source, oxygen concentration, the explosion space characteristics, and external environment conditions. Different factors play different roles in different periods and different states.

2. The gas explosion in coal mines can cause great destruction; the injury effect on personnel is mainly manifested in three aspects: the killing effect of shock wave overpressure (impulse) on personnel; the injury caused by dynamic pressure; the personnel asphyxiation effect caused by toxic and harmful gases. The three kinds of personnel injury effect act individually or simultaneously.

3. For gas explosion in blind roadway, the impaction of the dynamic pressure impact of shock wave and asphyxiation of toxic and harmful gases is relatively small, thus overpressure (impulse) of shock wave is mainly considered to measure damage caused by gas explosion. The damage of shock wave overpressure can be described by three characteristic parameters: peak overpressure, impulse and duration time.

4. The failure process of gas explosion in coal mines can be described as follows: firstly, the compression stress of shock wave makes the coal and rock of roadway wall compressed and broken, resulting in compression cracks and initial cracks instantly; secondly, the radial tension stress and reflection tension stress of stress wave make cracks on the wall extend and causes the wall broken more seriously, including the expansion of initial cracks and the formation of secondary cracks; and then, the expansion of explosion gas makes cracks in the roadway wall intersect to form block, the size of broken area increases, making the roadway wall broken more seriously.

\section{Acknowledgements}

None.

\section{Conflict of interest}

The Author declares there is no conflict of interest.

\section{References}

1. Cao FX. Study on multi-damage effect of explosion. Master's Thesis, Nanjing University of Science and Technology, China; 2008.

2. Zhong Q. Study on assessment methods of damage effect of explosionhazard-source. Master's Thesis, Nanjing University of Science and Technology, China; 2007.

3. Qu ZM. Study on destructive effect of gas explosion in coal mines. In: Chinese Conference of Theoretical and Applied Mechanics2005(CCTAM’2005). Beijing: Science Press, 2005;35-37.

4. Yang SZ, Jing GX, Jia ZW. Injury study on impact current of gas explosion in coal mine. Journal of China Coal Society. 2009;34(10):1354-1357.

5. Ye Q. The study on the character of gas explosion propagation and suppression technology by porous materials. Doctor's Thesis, China University of Mining and Technology. 2007. 
6. Gun H, Guang ZY. Effect of gas pressure on the transport properties of outburst-prone coal. Disaster Advances. 2011;4(3):20-23.

7. Ye Q, Lin BQ, Jian CG, et al. Propagation characteristics of gas explosion in duct with sharp change of cross sections. Disaster Advances. 2012;5(4):999-10003.
8. Lin BQ, Ye Q. Mechanism and control technology for gas explosion in coal mines. Xuzhou: China University of Science and Technology Press; 2012.

9. Xiong DY, Gu YC. New progress of theory and technology of rock blasting. Beijing: Metallurgical Industry Press; 2002. 